\title{
MRI shows thickening and altered diffusion in the median and ulnar nerves in multifocal motor neuropathy
}

\author{
Wieke Haakma $^{1,2}$ (1) $\cdot$ Bas A. Jongbloed ${ }^{3} \cdot$ Martijn Froeling $^{1} \cdot$ H. Stephan Goedee ${ }^{3} \cdot$ \\ Clemens Bos $^{1} \cdot$ Alexander Leemans $^{4} \cdot$ Leonard H. van den Berg $^{3} \cdot$ Jeroen Hendrikse $^{1}$ • \\ W. Ludo van der $\mathrm{Pol}^{3}$
}

Received: 18 April 2016/Revised: 9 August 2016/Accepted: 22 August 2016 / Published online: 21 September 2016

(C) The Author(s) 2016. This article is published with open access at Springerlink.com

\begin{abstract}
Objectives To study disease mechanisms in multifocal motor neuropathy (MMN) with magnetic resonance imaging (MRI) and diffusion tensor imaging (DTI) of the median and ulnar nerves.

Methods We enrolled ten MMN patients, ten patients with amyotrophic lateral sclerosis (ALS) and ten healthy controls (HCs). Patients underwent MRI (in a prone position) and nerve conduction studies. DTI and fat-suppressed T2-weighted scans of the forearms were performed on a 3.0T MRI scanner. Fibre tractography of the median and ulnar nerves was performed to extract diffusion parameters: fractional anisotropy (FA), mean (MD), axial (AD) and radial (RD) diffusivity. Cross-sectional areas (CSA) were measured on T2-weighted scans.

Results Forty-five out of 60 arms were included in the analysis. AD was significantly lower in MMN patients $\left(2.20 \pm 0.12 \times 10^{-3} \mathrm{~mm}^{2} / \mathrm{s}\right)$ compared to ALS patients
\end{abstract}

Electronic supplementary material The online version of this article (doi:10.1007/s00330-016-4575-0) contains supplementary material, which is available to authorized users.

Wieke Haakma

w.haakma@umcutrecht.nl

1 Department of Radiology, University Medical Center Utrecht, Utrecht, The Netherlands

2 Department of Forensic Medicine and Comparative Medicine Lab, Aarhus University, Aarhus, Denmark

3 Brain Centre Rudolf Magnus, Department of Neurology and Neurosurgery, University Medical Center Utrecht, Utrecht, The Netherlands

4 Image Sciences Institute, University Medical Center Utrecht, Utrecht, The Netherlands $\left(2.31 \pm 0.17 \times 10^{-3} \mathrm{~mm}^{2} / \mathrm{s} ; p<0.05\right)$ and $\mathrm{HCs}(2.31$ $\left.\pm 0.17 \times 10^{-3} \mathrm{~mm}^{2} / \mathrm{s} ; p<0.05\right)$. Segmental analysis showed significant restriction of $\mathrm{AD}, \mathrm{RD}$ and $\mathrm{MD}(p<0.005)$ in the proximal third of the nerves. CSA was significantly larger in MMN patients compared to ALS patients and HCs $(p<0.01)$.

Conclusions Thickening of nerves is compatible with changes in the myelin sheath structure, whereas lowered $\mathrm{AD}$ values suggest axonal dysfunction. These findings suggest that myelin and axons are diffusely involved in MMN pathogenesis. Key Points

- Diffusion magnetic resonance imaging provides quantitative information about multifocal motor neuropathy (MMN).

- Diffusion tensor imaging allows non-invasive evaluation of the forearm nerves in $M M N$.

- Nerve thickening and lowered diffusion parameters suggests myelin and axonal changes.

- This study can help to provide insight into pathological mechanisms of MMN.

Keywords Magnetic resonance imaging $\cdot$ Diffusion tensor imaging $\cdot$ Multifocal motor neuropathy $\cdot$ Amyotrophic lateral sclerosis $\cdot$ Median and ulnar nerve

\section{Introduction}

Multifocal motor neuropathy (MMN) is a rare disorder characterized by progressive, asymmetric and predominantly distal limb weakness without any sensory involvement [1]. The diagnosis of MMN is mainly based on the combination of clinical characteristics and specific nerve conduction abnormalities, i.e. conduction block. MMN is a mimic of the early phases of amyotrophic lateral sclerosis (ALS) and progressive muscular atrophy from which it needs to be distinguished, 
since the prognosis of MMN is much better than that of motor neuron disorders. Although patients with MMN respond to treatment with intravenous or subcutaneous immunoglobulins [2-4], progressive weakness of arms and hands due to accumulating axonal damage causes severe disability in a subgroup of patients $[1,5]$.

The pathogenic mechanisms that underlie MMN are incompletely understood. The presence of anti-GM1 IgM antibodies in more than half of the patients may suggest that MMN is caused by anti-GM1 antibody-mediated damage at or in the proximity of the nodes of Ranvier. This likely plays a role in the phenomenon of conduction block and in the demyelination or disruption of the compact myelin structure [2, 6]. Demyelination or disruption of the compact myelin structure represents an alternative pathogenic mechanism that causes MMN [6]. There are few pathological studies of affected motor nerves [7, 8], and there are no animal models for MMN. There is a need for new methodology to elucidate MMN pathogenesis and to eventually improve treatment strategies.

Magnetic resonance imaging (MRI) can be used to study the brachial plexus and peripheral nerves $[9,10]$. MRI T1- and T2-weighted images can provide anatomical detail and diffusion tensor imaging (DTI) technique information on the microstructural organization of nervous tissue [11-13] and peripheral nerves [14-17]. This unique combination may help to identify relevant disease mechanisms in patients with MMN. In this study we therefore used MRI and DTI to visualize the median and ulnar nerves in the forearm of patients with MMN and ALS and in healthy controls.

\section{Materials and methods}

\section{Patient characteristics}

We enrolled ten patients with MMN, ten patients with ALS and ten healthy controls at the neuromuscular outpatient clinic of the University Medical Centre Utrecht, a tertiary referral centre for neuromuscular disorders. Patients with MMN and ALS fulfilled diagnostic consensus criteria for definite or probable MMN and the El Escorial criteria for ALS, respectively $[4,18]$. All patients with ALS had clinical signs of lower motor neuron involvement (i.e. weakness, atrophy and/or fasciculations) in the forearm or hand. Patients and healthy controls were matched for age and gender. All patients with MMN were on immunoglobulin maintenance treatment and all patients with ALS used riluzole. All patients and healthy controls underwent a standardized clinical examination including muscle strength testing of the wrist, thumb and finger flexion, opponens pollicis, abductor pollicis brevis, finger spreading and adductor pollicis, together with sensory testing. Clinical examinations, electromyogram and MRI studies were performed on the same day. The local institutional review board approved this study and we obtained written informed consent from each subject prior to inclusion.

\section{Nerve conduction study protocol}

Nerve conduction studies were performed using a Nicolet VIKING IV electromyogram machine (CareFusion, Tokyo, Japan) after the limbs were warmed in water at $37^{\circ} \mathrm{C}$ for $30 \mathrm{~min}$. One of us (SG) was unaware of the clinical diagnosis and performed nerve conduction studies using a shortened version of a previously published protocol [4], consisting of motor nerve stimulation of the median nerve (recording $\mathrm{m}$. abductor pollicis brevis) and ulnar nerve (recording $\mathrm{m}$. abductor digiti $\mathrm{V}$ ) on both sides up to the axilla. We used the definition of conduction block as described in the diagnostic consensus criteria for MMN [18]. Axonal loss was defined as a decrease of distal compound muscle action potential (CMAP) below to 2 standard deviations of the lower limit of normal, i.e. a CMAP $<3.5 \mathrm{mV}$ for the median nerve and a distal CMAP of $<2.8 \mathrm{mV}$ for the ulnar nerve.

\section{MRI protocol and data acquisition}

All subjects underwent MRI of both forearms. Scans were acquired on a 3 Tesla MR system (Achieva, Philips Healthcare, Best, The Netherlands) with a 32-channel phased-array surface coil. Patients were positioned in a prone position with one arm placed above the head as described previously [19]. Patients were repositioned when the other arm was scanned. DTI was performed based on diffusionweighted spin echo single-shot echo planar imaging in the axial plane with the following parameters: $\mathrm{TE}=66 \mathrm{~ms}$, $\mathrm{TR}=6,340 \mathrm{~ms}$, SENSE factor 2 , FOV $240 \times 120 \mathrm{~mm}^{2}$, matrix size $160 \times 80,60$ slices with thickness $=4.0 \mathrm{~mm}$, resulting in a voxel size of $1.5 \times 1.5 \times 4.0 \mathrm{~mm}^{3}$, half scan 0.69 , SPIR fat suppression, b-values 0 , and $800 \mathrm{~s} / \mathrm{mm}^{2}, \mathrm{NSA}=1$, and 15 gradient directions. The total acquisition time was 9:32 min. As an anatomical reference, axial fat-suppressed T2-weighted scans were acquired with the following parameters: $\mathrm{TE}=90 \mathrm{~ms}, \mathrm{TR}=7139 \mathrm{~ms}$, SENSE factor 1.5 , FOV $120 \times 120 \mathrm{~mm}^{2}$, matrix size $240 \times 234$, slices with thickness of $4.0 \mathrm{~mm}$, and spectral attenuated inversion-recovery fat suppression. One stack was used with 60 slices for both the DTI and the T2-weighted scan. Scans with low quality, evaluated by visual inspection, for example due to movement, were excluded from analysis.

\section{DTI processing}

The DTI data were processed using ExploreDTI (www. ExploreDTI.com) [20]. Images were corrected for subject motion, eddy current-induced distortions and susceptibility 
artefacts [21, 22]. Diffusion tensors were calculated using the REKINDLE method [23] and diffusion parameters were subsequently obtained, which consisted of (1) the fractional anisotropy (FA), (2) the mean diffusivity (MD), (3) the axial diffusivity (AD), and (4) the radial diffusivity (RD) [14]. A standardized deterministic streamline approach was used to reconstruct the fibre tract [24].

\section{Fibre tractography and diffusion parameters}

To visualize the nerves and extract diffusion parameters, tractography was used. FA range was set to $0.1-0.9$, step size of $1 \mathrm{~mm}$, minimum fibre length was set to $100 \mathrm{~mm}$, and the fibre angle was set to $30^{\circ}$ per integration step. Fibre tracts, generated by whole volume seeding $\left(1.5 \times 1.5 \times 1.5 \mathrm{~mm}^{3}\right)$, belonging to the forearm nerves were selected by placing 'AND' region of interests (ROIs) in four locations in the arm: at the level of the pronator quadratus, one-third of the ulna, twothirds of the ulna, and the junction of the supinator with the radius [16], as shown in Fig. 1. These locations were chosen as they are relatively easy to locate and therefore provide a reproducible way of selecting the ROIs in each patient in the same way across subjects. 'AND' ROIs only select those tracts that run through all the 'AND' ROIs. This means that from all the fibre tracts generated from whole volume seeding, only those are selected that span all four predefined locations in the arm. Resulting tracts were used to calculate the diffusion parameters.

To investigate if DTI parameters were biased by the number of tracts and tract length [25], and to investigate to what extent diffusion metrics are distributed homogeneously along the nerve, additional analyses of shorter nerve segments were performed. For this purpose the forearm was subdivided into three segments defined by the ROI positions as described above and shown in Fig. 1. Tracts were selected for each of these individual subsections defined by only two of the four 'AND' ROIs and diffusion parameters were calculated for each of the three segments individually.

\section{Cross-sectional areas}

Nerve cross-sectional area (CSA) was assessed on T2weighted scans, at the predefined four locations in the arm: at the level of the pronator quadratus, one-third of the ulna, two-thirds of the ulna, and the junction of the supinator with the radius as shown in Fig. 1. The mean CSA of each nerve was then calculated based on the average CSA of these four locations.

\section{Statistical analysis}

We used SPSS version 20.0 (SPSS Inc., Chicago, IL, USA) for statistical analysis. A general linear model was used to compare the DTI parameters and the CSA measurements between the three groups. We used Bonferroni correction to correct for multiple testing. The analysis was performed for the whole nerve segment as well as for the individual segments and was based on both median and ulnar nerves. Correction for clustering of the data of scanning two arms in one patient was included into the model, and sex and age were taken into account [13]. Pearson correlation was used to check for correlation between the CSA and the diffusion parameters, and between duration of symptoms and diffusion parameters, where $p<0.05$ was considered to be significant.

\section{Results}

\section{Patient characteristics and nerve conduction studies}

Patient characteristics are summarized in Table 1. There was a significant difference in duration of symptoms $(p<0.001)$ between patients with MMN and ALS. Distribution and severity of weakness was similar in both patient groups. Nerve conduction studies showed conduction block in seven out of $40(18 \%)$ nerves, all in patients with MMN. Distal CMAP amplitudes were consistent with axonal loss.

\section{MRI protocol and data acquisition}

Data quality in patients was lower than in healthy controls. Based on visual inspection, DTI images of 15 out of 60 arms (seven arms of MMN patients, six arms of ALS patients and two arms of healthy controls) had to be excluded due to motion distortion or other MR-related problems resulting in a total of 45 scans of arms that were available for analysis. T2-weighted scans of nine out of 60 arms (three arms of MMN patients, four arms of ALS patients and two arms of healthy controls) had to be excluded, resulting in a total of 51 arms that were used for analysis.

\section{Fibre tractography}

The median and ulnar nerves could be reconstructed with fibre tractography in 40 of the 45 datasets. Figure 2 shows the tracts derived from the median and ulnar nerves in patients with MMN and ALS as well as in a healthy control. In five datasets tracts could not be reconstructed in seven nerves (six nerves of four MMN patients, and one nerve in a healthy control).

In total four nerves with conduction blocks and three nerves with axonal damage remained for analyses in the MMN group, and five nerves with axonal damage in the ALS group. 


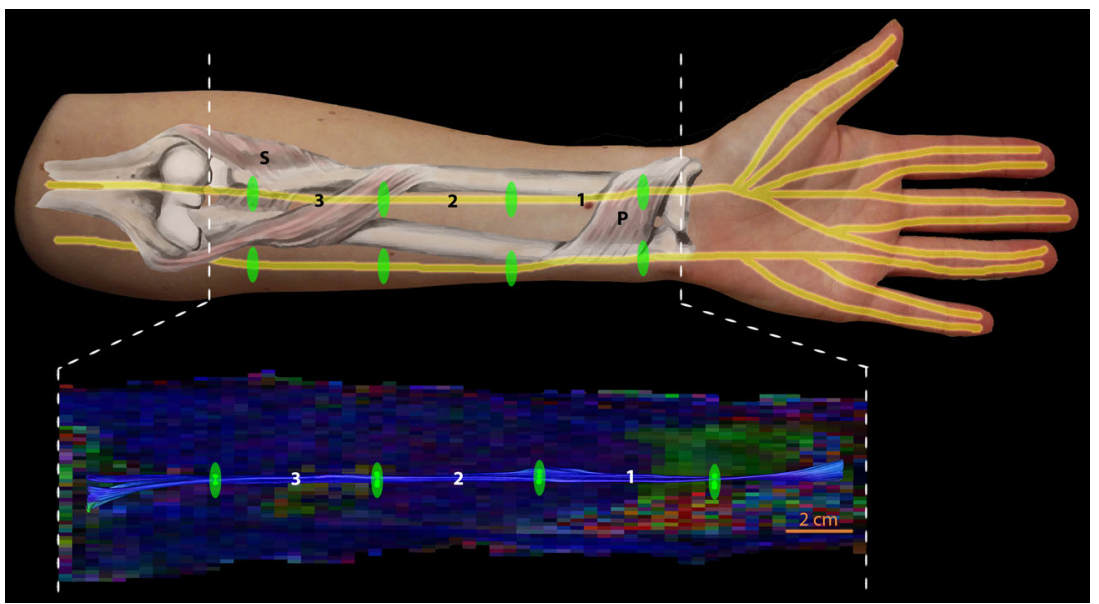

Fig. 1 Overview of the region of interest (ROI) positioning along the nerves in the arm (upper image) and the colour-encoded DTI (lower image), where green indicates anterior-posterior, red indicates left-right and blue indicates inferior-superior. The first ROI was placed at the pronator quadratus $(\mathrm{P})$, and the second and third ROIs at one-third and two-thirds of the ulna, respectively, and the fourth ROI was placed at the location of the junction of the supinator (S) with the radius. The tracts were analysed along the entire segment, and segments 1, 2, and 3 individually
Supplementary Table 1 shows the number of tracts per nerve and nerve segment. It was not possible to find tracts in three nerves of segments 1 and 3 and in two nerves of segment 2 in patients with MMN, and in one nerve in segment 1 in a healthy control.

\section{Diffusion parameters}

There were no significant differences between the two arms. Therefore, left and right arms were combined in the data analysis. The calculated average diffusion parameters of all tracts belonging to the median and ulnar nerves are summarized in Table 2. There was a significant difference in AD of nerves of patients with MMN $\left(2.20 \pm 0.12 \times 10^{-3} \mathrm{~mm}^{2} / \mathrm{s}\right)$ compared to patients with $\operatorname{ALS}\left(2.31 \pm 0.17 \times 10^{-3} \mathrm{~mm}^{2} / \mathrm{s} ; p<0.05\right)$ and to healthy controls $\left(2.31 \pm 0.17 \times 10^{-3} \mathrm{~mm}^{2} / \mathrm{s} ; p<0.05\right)$. There were no significant differences in FA, MD and RD between the groups.

Results of diffusion parameters of nerves with and without reduced distal CMAP are shown in Table 3. Nerves with reduced distal CMAP (both MMN and ALS patients) showed lower MD and RD values $(p<0.05)$. The $\mathrm{AD}$ in these nerves showed a tendency (not significant $p=0.083$ ) towards lower values.

Segmental analysis of diffusion parameters also showed a significantly lower $A D$ value in segment 3 for MMN patients compared to ALS patients $(p<0.005)$ and healthy controls $(p<0.05)$, and additionally significantly lower MD and RD $(p<0.005)$ values in patients with MMN compared to those with ALS, as shown in Table 2.
Table 1 Characteristics of patients with multifocal motor neuropathy (MMN), amyotrophic lateral sclerosis (ALS) and healthy controls

\begin{tabular}{lccc}
\hline & MMN $(n=10)$ & ALS $(n=10)$ & $\begin{array}{c}\text { Healthy controls } \\
(n=10)\end{array}$ \\
\hline $\begin{array}{l}\text { Mean age, years (range) } \\
\text { Male (\%) }\end{array}$ & $54(29-67)$ & $53(40-60)$ & $54(29-67)$ \\
$\begin{array}{l}\text { Median duration of symptoms } \\
\text { in months (range) }\end{array}$ & $82(80 \%)$ & $8(80 \%)$ & $8(80 \%)$ \\
$\begin{array}{l}\text { Median duration of treatment } \\
\text { in months (range) }\end{array}$ & $12(1-39)$ & $11(6-34) *$ & - \\
$\begin{array}{l}\text { Weakness lower arm (\%) } \\
\text { Number of conduction blocks }(\%)\end{array}$ & $15 / 20(75 \%)$ & $4(1-24)$ & - \\
$\begin{array}{l}\text { Number of nerves with distal } \\
\text { compound muscle action } \\
\text { potential }<\text { lower limit of } \\
\text { normal }(\%)\end{array}$ & $7(18 \%)$ & $0(0 \%)$ & $0(0 \%)$ \\
\hline
\end{tabular}

$* P<0.001$ 
Fig. 2 Fibre tractography of the median and ulnar nerve in a multifocal motor neuropathy (MMN) patient, amyotrophic lateral sclerosis (ALS) patient and healthy control (HC). The colourencoding is according to the axial diffusivity (in units $\mathrm{mm}^{2} / \mathrm{s}$ )

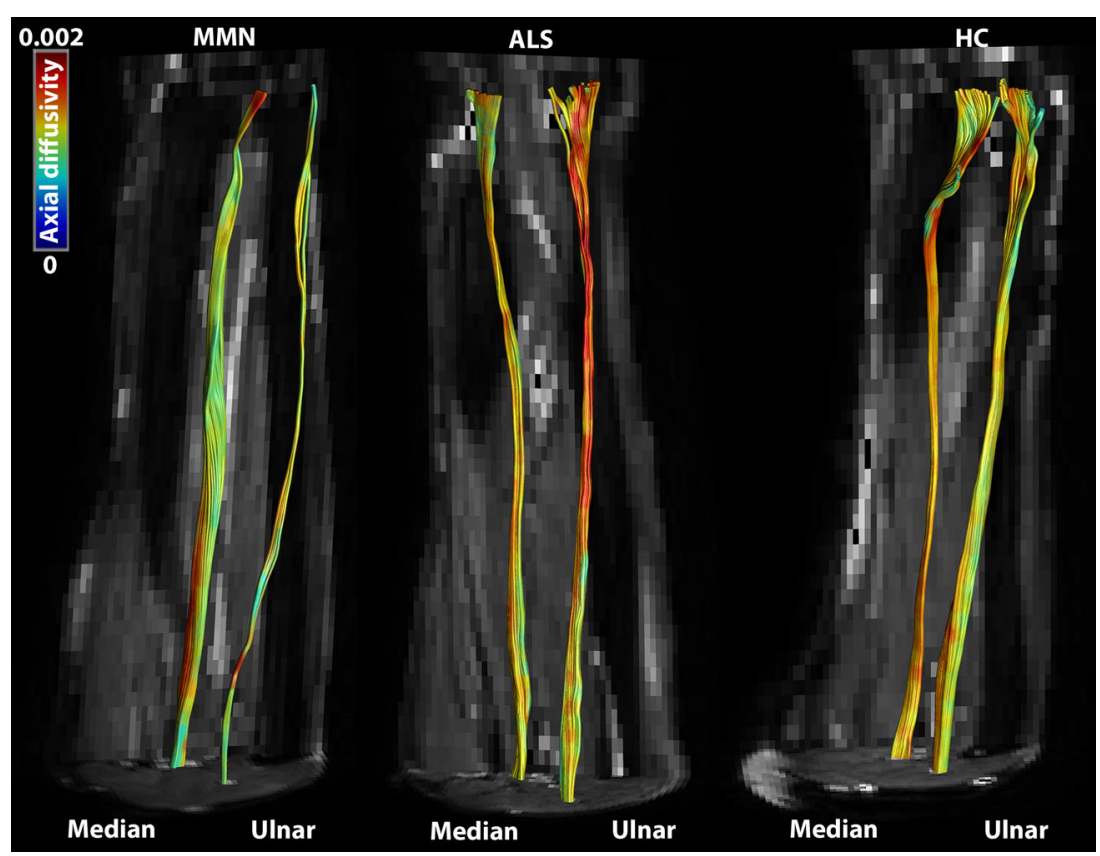

\section{Cross-sectional areas}

Figure 3 shows representative examples of CSA of the median nerve of a patient with MMN, a patient with ALS and a healthy control on T2-weighted scans. The mean CSA of the median and ulnar nerves on T2-weighted scans were significantly larger in patients with $\mathrm{MMN}$ (median $=9.40$ $\pm 2.87 \mathrm{~mm}^{2}$, and ulnar $=7.06 \pm 1.84 \mathrm{~mm}^{2}$ ) compared to those with ALS (median $=7.23 \pm 1.47 \mathrm{~mm}^{2}$, and ulnar $=5.68$ $\pm 0.93 \mathrm{~mm}^{2}$ ) and to healthy controls (median $=6.88$ $\pm 1.41 \mathrm{~mm}^{2}$, and ulnar $=5.36 \pm 0.89 \mathrm{~mm}^{2}$ ) (Fig. 4). There was no correlation between CSA and any of the diffusion parameters (FA, MD, AD and $\mathrm{RD})(\max r=0.262)$, or between duration of symptoms and diffusion parameters ( $\max$ $r=0.391$ ). Nerves with reduced CMAP amplitudes did not show a significant difference in CSA compared to nerves with normal CMAP amplitudes (see Table 3). There was no correlation between the CSA and any of the diffusion parameters ( $\max r=0.160)$ in the nerves with reduced CMAP amplitudes.

\section{Discussion}

This MRI study shows anatomical and diffusion abnormalities in peripheral nerves from patients with MMN. We found significant nerve enlargement in combination with a significant reduction of axial diffusion. These in vivo results suggest that pathogenic mechanisms in MMN might affect both the axon and myelin sheath, as was earlier suggested by pathological abnormalities near the site of conduction block [8].

The median and ulnar nerves of MMN patients were 25$30 \%$ larger than those in the healthy controls and ALS patients. The diffuse rather than focal nerve enlargements are in line with high resolution ultrasound studies of peripheral nerves $[26,27]$ and the brachial plexus $[9,28]$. Pathogenic mechanisms underlying MMN therefore seem to affect significant lengths of motor nerves rather than patchy and focal involvement, as suggested by the observed patterns of weakness and conduction block $[1,5]$.

There are clear indications that nerve thickening on MRI reflects involvement of the myelin sheath. It is a consistent feature of both genetic and acquired demyelinating polyneuropathies, i.e. Charcot-Marie-Tooth type 1 and chronic inflammatory demyelinating polyneuropathy $[28,29]$. This is further supported by the occasional pathological observation of onion bulb formation in nerve biopsy studies in MMN $[7,30,31]$.

Demyelination is probably not the only pathological mechanism that underlies MMN, since it does not explain all disease characteristics, such as the phenomenon of cold paresis [32]. Findings in the rabbit model for acute motor axonal neuropathy and human motor neuron model for MMN $[33,34]$ and clinical observations of significant axonal damage in patients with MMN [5, 35] suggest additional pathological mechanisms that directly affect the axon [36]. The DTI findings in this study, in particular the reduced AD values, support this concept. Reduced $\mathrm{AD}$ values reflect pathological changes that impair diffusion in the length of the axon and are associated with Wallerian degeneration in animal studies [11, 12, 37]. In a recently developed in vitro model of anti-GM1 IgM antibodymediated damage to human motor nerves, we observed focal widening of the axon that preceded Wallerian degeneration [34]. MRI studies in an ischaemia-model of rat sciatic nerve showed that this process of axonal 'beading' was associated 
Table 2 Mean diffusion parameters (fractional anisotropy (FA), mean (MD), axial (AD) and radial (RD) diffusivity) with standard deviation (SD) based on both median and ulnar nerves in patients with multifocal motor neuropathy (MMN), amyotrophic lateral sclerosis (ALS) and healthy controls (HC)

\begin{tabular}{llll}
\hline & MMN & ALS & HC \\
\hline FA & & & \\
Entire nerve $^{\mathrm{a}}$ & $0.44 \pm 0.04$ & $0.43 \pm 0.05$ & $0.44 \pm 0.04$ \\
${\text { Segment } 1^{\mathrm{b}}}^{\mathrm{c}}$ & $0.43 \pm 0.05$ & $0.43 \pm 0.05$ & $0.43 \pm 0.04$ \\
Segment $^{\mathrm{c}}$ & $0.44 \pm 0.05$ & $0.43 \pm 0.05$ & $0.44 \pm 0.05$ \\
Segment $3^{\mathrm{d}}$ & $0.46 \pm 0.06$ & $0.44 \pm 0.03$ & $0.45 \pm 0.04$ \\
$\mathrm{MD}\left(\times 10^{-3} \mathrm{~mm}^{2} / \mathrm{s}\right)$ & & & \\
Entire nerve & $1.44 \pm 0.10$ & $1.52 \pm 0.15$ & $1.51 \pm 0.14$ \\
Segment 1 & $1.50 \pm 0.18$ & $1.46 \pm 0.17$ & $1.51 \pm 0.16$ \\
Segment 2 & $1.43 \pm 0.16$ & $1.49 \pm 0.18$ & $1.49 \pm 0.14$ \\
Segment 3 & $1.38 \pm 0.14^{* *}$ & $1.50 \pm 0.10^{* *}$ & $1.45 \pm 0.11$ \\
AD $\left(\times 10^{-3} \mathrm{~mm}^{2} / \mathrm{s}\right)$ & & & \\
Entire nerve & $2.20 \pm 0.12^{*}$ & $2.31 \pm 0.17^{*}$ & $2.31 \pm 0.17^{*}$ \\
Segment 1 & $2.26 \pm 0.22$ & $2.22 \pm 0.20$ & $2.29 \pm 0.20$ \\
Segment 2 & $2.19 \pm 0.18$ & $2.27 \pm 0.22$ & $2.27 \pm 0.15$ \\
Segment 3 & $2.16 \pm 0.18^{*}$ & $2.30 \pm 0.13^{*}$ & $2.25 \pm 0.14^{*}$ \\
RD $\left(\times 10^{-3} \mathrm{~mm}^{2} / \mathrm{s}\right)$ & & & \\
Entire nerve & $1.06 \pm 0.10$ & $1.13 \pm 0.15$ & $1.11 \pm 0.14$ \\
Segment 1 & $1.12 \pm 0.18$ & $1.08 \pm 0.16$ & $1.11 \pm 0.15$ \\
Segment 2 & $1.05 \pm 0.16$ & $1.11 \pm 0.18$ & $1.09 \pm 0.14$ \\
Segment 3 & $0.99 \pm 0.14^{* *}$ & $1.11 \pm 0.09^{* *}$ & $1.05 \pm 0.11$ \\
\hline
\end{tabular}

${ }^{\text {a }}$ Whole segment: $n=20, n=28$ and $n=36$ for, respectively, MMN, ALS and $\mathrm{HC}$

${ }^{\mathrm{b}}$ Segment 1: $n=23, n=28$ and $n=35$ for, respectively, MMN, ALS and HC

${ }^{\mathrm{c}}$ Segment 2: $n=24, n=28$ and $n=36$ for, respectively, MMN, ALS and HC

${ }^{\mathrm{d}}$ Segment 3: $n=23, n=28$ and $n=36$ for, respectively, MMN, ALS and HC

* Significant difference in MMN vs. ALS and controls $(\mathrm{p}<0.05)$

** Significant difference in MMN vs. ALS $(\mathrm{p}<0.005)$

Table 3 Mean diffusion parameters and cross-sectional areas (CSA) of the median and ulnar nerves with reduced compound muscle action potential (CMAP) amplitudes, i.e. smaller than the lower limit of normal (LLN) reflecting axonal loss, versus nerves with normal CMAP amplitudes

\begin{tabular}{lll}
\hline & CMAP < LLN $(N=8)$ & CMAP $>$ LLN $(N=76)$ \\
\hline FA & $0.47 \pm 0.05^{*}$ & $0.44 \pm 0.04^{*}$ \\
$\mathrm{MD}\left(\times 10^{-3} \mathrm{~mm}^{2} / \mathrm{s}\right)$ & $1.40 \pm 0.12^{*}$ & $1.51 \pm 0.14^{*}$ \\
$\mathrm{AD}\left(\times 10^{-3} \mathrm{~mm}^{2} / \mathrm{s}\right)$ & $2.19 \pm 0.14$ & $2.30 \pm 0.16$ \\
$\mathrm{RD}\left(\times 10^{-3} \mathrm{~mm}^{2} / \mathrm{s}\right)$ & $1.00 \pm 0.13^{*}$ & $1.12 \pm 0.14^{*}$ \\
$\mathrm{CSA}$ & $7.09 \pm 1.29$ & $6.48 \pm 1.37$ \\
\hline
\end{tabular}

$* p<0.05$

$F A$ fractional anisotropy, $M D$ mean diffusivity, $A D$ axial diffusivity, $R D$ radial diffusivity with significantly restricted $\mathrm{AD}$ and virtually unchanged $\mathrm{RD}$ and FA values [38]. The reduced AD values may therefore reflect pathological changes in motor axons of patients with MMN. The subanalysis performed on nerves with reduced CMAP amplitudes shows lower FA, MD and RD values and a tendency toward lower AD in the median and ulnar nerves. This tendency toward lower AD could be associated with a reduction of axon integrity [13]. Reduced MD might be due to disruption of the cytoskeleton, increasing the viscosity [39]. Detailed analysis of the association of conduction block and MRI and DTI abnormalities would be of added value to further explore the pathophysiological mechanisms behind MMN. However, this was not possible due to the low number of conduction blocks in this patient sample, which would make a statistical analysis severely underpowered. This is a topic for future larger scale studies.

Patients appeared more uncomfortable in the prone scanning position, and as a result motion artefacts were more common in patients than in healthy controls. This scanning position was a methodological limitation of this study and resulted in the exclusion of a significant number of scans due to the relatively low quality of this data. During development of the protocol we aimed to obtain a protocol with a sufficiently high resolution to distinguish the nerves and to have sufficient signal-to-noise ratio (SNR), as the SNR, amongst other things, will influence the precision of the DTI metrics [25, 40]. Future development of DTI protocols in the forearm should focus on the right trade-off in SNR, resolution (preferable $<1 \times 1 \mathrm{~mm}$ in plane), and scan time, as SNR and resolution will always come at the cost of increased scan time and thus patient discomfort [25]. Repositioning patients in the supine position and using dedicated arm coils could improve patient comfort and therefore reduce motion artefacts in future studies. This will improve data quality resulting in less data that need to be rejected due to artefacts.

We used a tract-based analysis approach with a minimum length of fibre tracts of $100 \mathrm{~mm}$ to exclude aberrant inclusion of muscle fibres. As a consequence, the number of tracts available for final analysis was small, since only a limited number of fibres can be traced over this range. Moreover, the error accumulation over a long tract range can become substantial and may introduce bias [25]. To overcome this problem, we additionally performed segmental analysis, in which smaller segments of the nerves were analysed resulting in more fibre tracts being included. The higher number of tracts allows for better sampling of the data. Segmental analysis showed similar AD changes and additional significant differences in RD values in the proximal segment (segment 3 ), but not in the distal segments (segments 1 and 2), of the median and ulnar nerves. Decreased RD values further support pathological processes that directly affect the axon, rather than demyelination $[11,41]$. 
Fig. 3 Axial plane of T2weighted scans of the forearm with the median $(\mathrm{M})$ and ulnar (U) nerves. a Multifocal motor neuropathy (MMN) patient with enlargement of the median nerve, (b) amyotrophic lateral sclerosis (ALS) patient and (c) healthy control

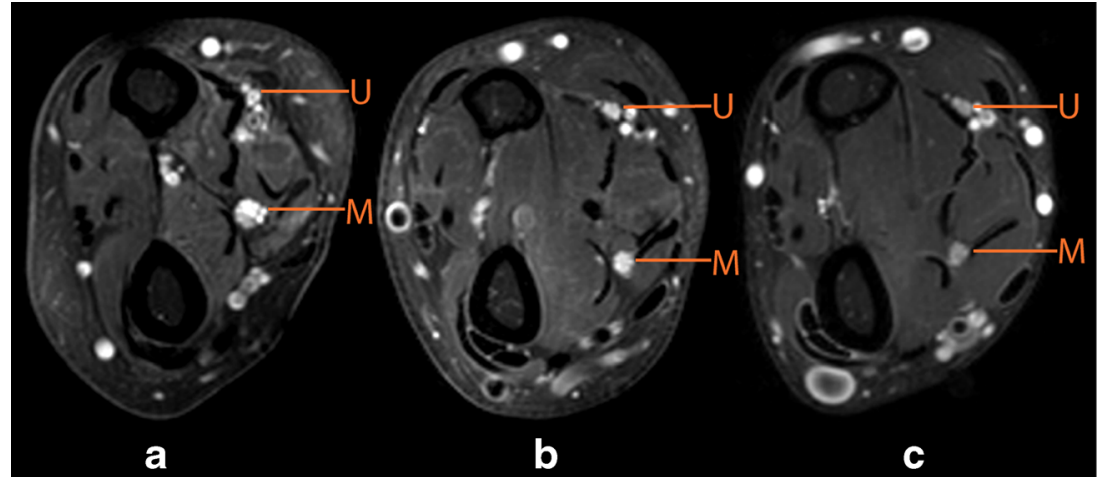

An obvious limitation of our study is the number of included patients, which limits its power. Furthermore, the age range of ALS patients did not fully match those of the MMN patients and healthy controls. As ALS and MMN are rare diseases, matching of these two groups is challenging. This was further complicated by the fact that we could only include a selection of ALS patients who were able to lie still in a prone position for a relatively long time. However, mean age, standard deviations and $95 \%$ confidence intervals of ALS patients were similar to those of patients with MMN and healthy controls.

In line with previous studies we found no differences in diffusion parameters between the left and right arms [42]. DTI has not been used extensively to investigate forearm nerves and there are few comparable studies of peripheral nerves of the arm in healthy controls [16] or patients with polyneuropathy [43, 44]. Comparison of previous results with our findings is further complicated by differences in MRI settings (e.g. smaller voxel size and higher b-value), the difference in threshold settings used for tractography (higher FA threshold results in higher FA values [25]) and patient characteristics [43].

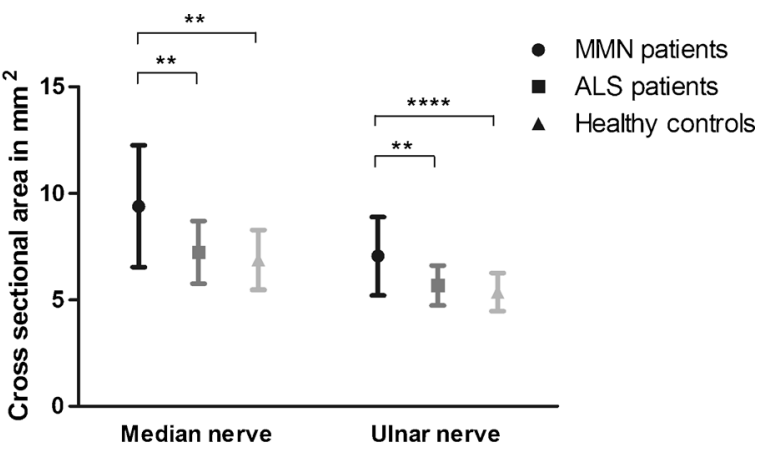

\footnotetext{
- MMN patients $\quad$ ALS patients $\quad \nabla$ Healthy controls ${ }^{*} \mathrm{p}<0.05,{ }^{* *} \mathrm{p}<0.01,{ }^{* * *} \mathrm{p}<0.001,{ }^{* * *} \mathrm{p}<0.0001$
}

Fig. 4 Cross-sectional area of the median and ulnar nerves in the forearm with standard deviation (SD). Multifocal motor neuropathy (MMN) patients differed significantly from amyotrophic lateral sclerosis (ALS) patients and healthy controls
Partial volume effects have to be considered when interpreting DTI results in small nerves [45]. Partial volume effects are caused by the voxels located on the edges of the nerves and thus partially contain muscle tissue signal, which has a lower $\mathrm{AD}$, and a higher $\mathrm{RD}$ than the nerve. As a consequence, partial volume effects would lower the AD and increase the RD values [19]. However, partial volume effects cannot explain the current findings of lower $\mathrm{AD}$ values as nerves of MMN patients had a larger CSA and consequently lower partial volume effects [45]. Partial volume effects could influence the RD values and provide an alternative explanation for the differences found in RD between patients with MMN and controls.

In conclusion, this study shows that MRI and DTI can detect lowered $\mathrm{AD}$ and enlarged CSA in patients with MMN compared to ALS and to healthy controls. These results can help to provide insight into pathological mechanisms of MMN. Future studies would be facilitated by improving patient comfort, for example through the use of dedicated arm coils and placing patients in a supine position, which could reduce motion artefacts and thus improve data quality. As a result less data need to be rejected due to artefacts. Comparative DTI studies of patients with MMN and other demyelinating peripheral nerve disorders, such as chronic inflammatory demyelinating polyneuropathy and Charcot-Marie-Tooth type 1A, could help to further clarify the aetiology of MMN.

Acknowledgments We would like to thank Niels Blanken (MRI radiographer, department of radiology) for his help in performing the MRI scans. We would like to thank Dirk Rutgers (radiologist) and Hessel Franssen (neurologist) for sharing their expert opinions on this topic. The scientific guarantor of this publication is Jeroen Hendrikse. The authors of this manuscript declare no relationships with any companies whose products or services may be related to the subject matter of the article. This study received funding as follows: H.S. Goedee receives research support from the Prinses Beatrix Spierfonds. A. Leemans receives research support of the VIDI Grant 639.072.411 from the Netherlands Organisation for Scientific Research (NWO). L.H. van den Berg serves on scientific advisory boards for the Prinses Beatrix Spierfonds, Thierry Latran Foundation, Biogen Idec and Cytokinetics; received an educational 
grant from Baxter International Inc.; serves on the editorial board of Amyotrophic Lateral Sclerosis and the Journal of Neurology, Neurosurgery and Psychiatry; and receives research support from the Prinses Beatrix Spierfonds, Netherlands ALS Foundation, The European Community's Health Seventh Framework Programme (grant agreement $n^{\circ}$ 259867), The Netherlands Organization for Health Research and Development (Vici Scheme, JPND (SOPHIA, STRENGTH)). J. Hendrikse receives support from the Netherlands Organization for Scientific Research (NWO) under grant $\mathrm{n}^{\circ} 91712322$, the European Research Council under grant agreements $n^{\circ} 637024$. W.L. van der Pol receives research support from the Prinses Beatrix Spierfonds; The Netherlands ALS Foundation; Stichting Spieren voor Spieren. One of the authors has significant statistical expertise. Institutional Review Board approval was obtained. Written informed consent was obtained from all subjects (patients) in this study. Methodology: case-control study, performed at one institution.

Open Access This article is distributed under the terms of the Creative Commons Attribution 4.0 International License (http:// creativecommons.org/licenses/by/4.0/), which permits unrestricted use, distribution, and reproduction in any medium, provided you give appropriate credit to the original author(s) and the source, provide a link to the Creative Commons license, and indicate if changes were made.

\section{References}

1. Cats EA, Van Der Pol WL, Piepers S et al (2010) Correlates of outcome and response to IVIg in 88 patients with multifocal motor neuropathy. Neurology 75:818-825

2. Vlam L, van der Pol W-L, Cats EA et al (2012) Multifocal motor neuropathy: diagnosis, pathogenesis and treatment strategies. Nat Rev Neurol 8:48-58

3. Nobile-Orazio E, Cappellari A, Meucci N et al (2002) Multifocal motor neuropathy: clinical and immunological features and response to IVIg in relation to the presence and degree of motor conduction block. J Neurol Neurosurg Psychiatry 72:761-766

4. Van den Berg-Vos RM, Franssen H, Wokke JH et al (2000) Multifocal motor neuropathy: diagnostic criteria that predict the response to immunoglobulin treatment. Ann Neurol 48:919-926

5. Van Asseldonk JTH, Van den Berg LH, Van den Berg-Vos RM et al (2003) Demyelination and axonal loss in multifocal motor neuropathy: distribution and relation to weakness. Brain 126:186-198

6. Susuki K, Rasband MN, Tohyama K et al (2007) Anti-GM1 antibodies cause complement-mediated disruption of sodium channel clusters in peripheral motor nerve fibers. J Neurosci 27:3956-3967

7. Kaji R, Oka N, Tsuji T et al (1993) Pathological findings at the site of conduction block in multifocal motor neuropathy. Ann Neurol $33: 152-158$

8. Taylor BV, Dyck PJB, Engelstad J et al (2004) Multifocal motor neuropathy: pathologic alterations at the site of conduction block. $\mathrm{J}$ Neuropathol Exp Neurol 63:129-137

9. Van Es HW, Van den Berg LH, Franssen H et al (1997) Magnetic resonance imaging of the brachial plexus in patients with multifocal motor neuropathy. Neurology 48:1218-1224

10. Chhabra A, Belzberg AJ, Rosson GD et al (2016) Impact of high resolution 3 tesla MR neurography (MRN) on diagnostic thinking and therapeutic patient management. Eur Radiol 26:1235-1244

11. Song S-K, Sun S-W, Ramsbottom MJ et al (2002) Dysmyelination revealed through MRI as increased radial (but unchanged axial) diffusion of water. Neuroimage 17:1429-1436
12. DeBoy CA, Zhang J, Dike S et al (2007) High resolution diffusion tensor imaging of axonal damage in focal inflammatory and demyelinating lesions in rat spinal cord. Brain 130:2199-2210

13. Heckel A, Weiler M, Xia A et al (2015) Peripheral nerve diffusion tensor imaging: assessment of axon and myelin sheath integrity. PLoS One 10:1-13

14. Haakma W, Dik P, Ten Haken B et al (2014) Diffusion tensor $\{\mathrm{MRI}\}$ and fiber tractography of the sacral plexus in children with spina bifida. J Urol 192:927-933

15. Barcelo C, Faruch M, Lapegue F et al (2013) 3-T MRI with diffusion tensor imaging and tractography of the median nerve. Eur Radiol 23:3124-3130

16. Zhou Y, Narayana PA, Kumaravel M et al (2014) High resolution diffusion tensor imaging of human nerves in forearm. J Magn Reson Imaging 39:1374-1383

17. Jengojan S, Kovar F, Breitenseher J et al (2015) Acute radial nerve entrapment at the spiral groove: detection by DTI-based neurography. Eur Radiol 25:1678-1683

18. Guideline PNSMMN (2010) European federation of neurological societies/peripheral nerve society guideline on management of multifocal motor neuropathy. Report of a joint task force of the european federation of neurological societies and the peripheral nerve society-first revisi. J Peripher Nerv Syst 15:295-301

19. Froeling M, Nederveen AJ, Heijtel DF et al (2012) Diffusion-tensor MRI reveals the complex muscle architecture of the human forearm. J Magn Reson Imaging 36:237-248

20. Leemans A, Jeurissen B, Sijbers J, Jones DK (2009) ExploreDTI: a graphical toolbox for processing, analyzing, and visualizing diffusion MR data. Proc Intl Soc Magn Reson Med 17:3536

21. Leemans A, Jones DK (2009) The B-matrix must be rotated when correcting for subject motion in DTI data. Magn Reson Med 61: 1336-1349

22. Irfanoglu MO, Walker L, Sarlls J et al (2012) Effects of image distortions originating from susceptibility variations and concomitant fields on diffusion MRI tractography results. Neuroimage 61: 275-288

23. Tax CMW, Otte WM, Viergever MA et al (2014) REKINDLE: robust extraction of kurtosis INDices with linear estimation. Magn Reson Med 73:794-808

24. Basser PJ, Pajevic S, Pierpaoli C et al (2000) In vivo fiber tractography using DT-MRI data. Magn Reson Med 44:625-632

25. Froeling M, Nederveen AJ, Nicolay K, Strijkers GJ (2013) DTI of human skeletal muscle: the effects of diffusion encoding parameters, signal-to-noise ratio and $\mathrm{T} 2$ on tensor indices and fiber tracts. NMR Biomed 26:1339-1352

26. Beekman R, van den Berg LH, Franssen H et al (2005) Ultrasonography shows extensive nerve enlargements in multifocal motor neuropathy. Neurology 65:305-307

27. Grimm A, Décard BF, Athanasopoulou I et al (2015) Nerve ultrasound for differentiation between amyotrophic lateral sclerosis and multifocal motor neuropathy. J Neurol 262:870-880

28. Shibuya K, Sugiyama A, Ito S et al (2014) Reconstruction magnetic resonance neurography in chronic inflammatory demyelinating polyneuropathy. Ann Neurol 77:1-5

29. Hiwatashi A, Togao O, Yamashita K et al (2016) Evaluation of chronic inflammatory demyelinating polyneuropathy: 3D nervesheath signal increased with inked rest-tissue rapid acquisition of relaxation enhancement imaging (3D SHINKEI). Eur Radiol

30. Corbo M, Abouzahr MK, Latov N et al (1997) Motor nerve biopsy studies in motor neuropathy and motor neuron disease. Muscle Nerve 20:15-21

31. Auer RN, Bell RB, Lee MA (1989) Neuropathy with onion bulb formations and pure motor manifestations. Can J Neurol Sci 16: 194-197 
32. Straver DCG, van Asseldonk J-TH, Notermans NC et al (2011) Cold paresis in multifocal motor neuropathy. J Neurol 258:212-217

33. Susuki K, Nishimoto Y, Yamada M et al (2003) Acute motor axonal neuropathy rabbit model: immune attack on nerve root axons. Ann Neurol 54:383-388

34. Harschnitz O, van den Berg LH, Johansen LE et al (2016) Autoantibody pathogenicity in a multifocal motor neuropathy iPSC-derived model. Ann Neurol 80:71-88

35. Van Asseldonk JTH, Van den Berg LH, Kalmijn S et al (2006) Axon loss is an important determinant of weakness in multifocal motor neuropathy. J Neurol Neurosurg Psychiatry 77: 743-747

36. Franssen H, Straver DCG (2014) Pathophysiology of immunemediated demyelinating neuropathies-Part II: Neurology. Muscle Nerve 49:4-20

37. Beaulieu C, Does MD, Snyder RE, Allen PS (1996) Changes in water diffusion due to Wallerian degeneration in peripheral nerve. Magn Reson Med 36:627-631

38. Budde MD, Frank JA (2010) Neurite beading is sufficient to decrease the apparent diffusion coefficient after ischemic stroke. Proc Natl Acad Sci U S A 107:14472-14477
39. Beaulieu C (2002) The basis of anisotropic water diffusion in the nervous system - a technical review. NMR Biomed $15: 435-455$

40. Jones DK (2010) Precision and accuracy in diffusion tensor magnetic resonance imaging. Top Magn Reson Imaging 21:87-99

41. Song SK, Yoshino J, Le TQ et al (2005) Demyelination increases radial diffusivity in corpus callosum of mouse brain. Neuroimage 26:132-140

42. Zhou Y, Kumaravel M, Patel VS et al (2012) Diffusion tensor imaging of forearm nerves in humans. J Magn Reson Imaging 36: 920-927

43. Breckwoldt MO, Stock C, Xia A et al (2015) Diffusion tensor imaging adds diagnostic accuracy in magnetic resonance neurography. Investig Radiol 50:498-504

44. Kakuda T, Fukuda H, Tanitame K et al (2011) Diffusion tensor imaging of peripheral nerve in patients with chronic inflammatory demyelinating polyradiculoneuropathy: a feasibility study. Neuroradiology 53:955-960

45. Vos SB, Jones DK, Viergever MA, Leemans A (2011) Partial volume effect as a hidden covariate in DTI analyses. Neuroimage 55: $1566-1576$ 